\title{
PSI Team
}

\author{
Alexander N. Kozhushkin \\ Program Systems Institute of Russian Academy of Sciences
}

\section{Introduction}

Our team - PSI was developed at Program Systems Institute of Russian Academy of Science. This paper is a short description of the dynamical refinement planning method that we use to construct our software agents.

Basic skills and roles of every agent are presented by means of the set of elementary plans. The purpose of the planning process is to compose the extended plan defining the behaviour of the agent from elementary ones.

The planning system (or just a planner), built in the agent, modifies extended plans depending on external conditions and the internal state of the agent. It adds new elementary plans to the extended one refining it and controls the execution of elementary plans in a body of the extended plan. Namely, planner can temporarily suspend (interrupt) execution of some elementary plan in favor of another one or abort execution of the inappropriate elementary plan. For its work planning system uses the family of basic relations (interruptability, priorities and plan levels) described below.

Although results of RoboCup'99 are not very well for the PSI team, we think that our method proved to be flexible and convenient for the programming the complex behaviours of the agents working in the unpredictable environment.

\section{Team Development}

Team Leader: Alexander N. Kozhushkin

Team Members:

Alexander N. Kozhushkin

- Program Systems Institute of Russian Academy of Sciences

- Russia

- Ph.D. student

- did attend the competition

Alexei P. Lisitsa

- Program Systems Institute of Russian Academy of Sciences

- Russia

- senior researcher

- did not attend the competition

Web page http://www.botik.ru/ soccer/ANK.html

M. Veloso, E. Pagello, and H. Kitano (Eds.): RoboCup-99, LNAI 1856, pp. 623-627, 2000.

Springer-Verlag Berlin Heidelberg 2000 


\section{Model of the planning system}

Consider a model of the planning system built in each player. It is defined by the discrete set of time moments $T=\{0,1,2,3, \ldots\}$, a set $O$ of elementary actions which soccer server can execute, a set $I$ of input (or external) states defined by all possible values of data available from soccer server and the space $\Omega$ of internal states of the player. $I \times T$ and $O$ represent input information (input) and actions (output) of the player, respectively. Play history, or just a play, from the point of view of a player may be defined as a map $H: T \rightarrow I \times \Omega$, where $H(t)$ consists of the information from soccer server and of the internal state of the player at the moment $t$. Let $B(i, \omega), C(i, \omega)$ be some logical conditions on the set of full states $I \times \Omega, \phi$ and $\psi$ be some maps, $\phi: I \times \Omega \rightarrow \Omega, \psi: I \times \Omega \rightarrow O$. Define elementary plan of the player as a tuple $p=\langle B, C, \phi, \psi\rangle$, where $B$ and $C$ are, respectively, its beginning and continuation conditions.

For our work we use some fixed finite set of basic elementary plans $\pi$. Define the family of subsets $D_{i} \subset \pi, i \in\left\{1,2, \ldots, n_{\text {lev }}\right\}$, where $n_{\text {lev }}>1$ the number of the hierarchic levels, such that $\cup_{i} D_{i}=\pi$ and $D_{i} \cap D_{j} \neq D_{i}$ for $i<j$. For every $D_{i}$ define partial order relation Prior $_{i}$ (priority). By means of plan hierarchic levels introduce the binary interruptability relation Int defined on $\pi \times \pi$, with the constraint on it: $\operatorname{Int}\left(p, p^{\prime}\right) \wedge p \in D_{i} \Rightarrow p^{\prime} \in D_{i+1}$. In the rest of the paper we suppose that if the set $\pi$ is defined then families $\left\{D_{i}\right\},\left\{\operatorname{Prior}_{i}\right\}$ and the Int relation are defined as well.

Explain the meaning of introduced notions. Each elementary plan from $\pi$ is designed to determine a skill or a role of the player or to solve a particular task. For example, there are plans designed to solve the task of the ball interception, overtaking another player and so on. Both $B$ and $C$ are applicability conditions of the plan. But in practice these conditions are different. The aim of the distinction between them is a making behaviour of agents more robust and stable.

The family $\left\{D_{i}\right\}$ i.e. plan levels determine the hierarchy on the plans. Levels divide all plans into main and auxiliary ones. Note, that same plan can fall onto different levels. This is in agreement with the intuition that same skill can be auxiliary to some main skill and, at the same time, auxiliary to another auxiliary one. The interruptability relation presents the further refinement concerning which plan can be suspended temporarily (interrupted) in favour of another one, auxiliary to it. The priority relations serve to resolve ambiguities in the cases when several elementary plans can be applied to the current situation. Thus $\left\{\right.$ Prior $\left._{i}\right\}$ relations play the role of evaluation functions.

For every time $t$ of the play $H$ there are two elementary plan subsets $A p p_{t}{ }^{H}=$ $\left\{p \in \pi \mid \models B_{p}(H(t))\right.$ and $C o n_{t}{ }^{H}=\left\{p \in \pi \mid \models C_{p}(H(t))\right\}$ of $\pi$ whose beginning and continuation conditions are satisfied at the moment $t$, respectively. We would like to name the set $A p p_{t}{ }^{H}$ as a set of applicable and $C o n_{t}{ }^{H}$ as a set of executable elementary plans.

Suppose that the set $\pi$ is defined, we build its extension $\pi^{*}$ by the following rules:

(i) $\pi \subset \pi^{*}$;

(ii) if $p=<B_{p}, C_{p}, \phi_{p}, \psi_{p}>\in \pi^{*}$ then plan $<C_{p}, C_{p}, \phi_{p}, \psi_{p}>$ is in $\pi^{*}$; 
(iii) if $p=<B_{p}, C_{p}, \phi_{p}, \psi_{p}>\in \pi^{*}$ and $p^{\prime}=<B_{p^{\prime}}, C_{p^{\prime}}, \phi_{p^{\prime}}, \psi_{p^{\prime}}>\in \pi^{*}$ then plan $<B_{p} \wedge C_{p^{\prime}}, C_{p} \wedge C_{p^{\prime}}, \phi_{p}\left(i, \phi_{p^{\prime}}(i, \omega)\right), \psi_{p}>$ is in $\pi^{*}$, where $i \in I, \omega \in \Omega$.

Thus $\pi^{*}$ the set of all elementary plans which an agent can use, "tuning" the basic ones to current conditions.

We define extended plan $P$ as a word of a plan language $L$ in the alphabet $A=\left(\pi^{*} \cup\{*\}\right) \times(\pi \cup\{*\}) \times\left\{0, \ldots, n_{l e v}\right\}$. We use the symbol "*" as a abbreviation of the $(*, *, 0)$. $L$ is defined by the next rules (where $Q, Q_{1}$ are letter sequences without "*", possibly empty):

1. $* \in L$;

2. if $Q * \in L$ and $p \in D_{1}$, then $Q *(p, p, 1) \in L$;

3. if $Q * l Q_{1} \in L$ where $l \in \pi * \times \pi \times\left\{0, \ldots, n_{l e v}\right\}$ and $p \in \pi^{*}$ then $Q l * Q_{1} \in L$; 4. if $Q *(<B, C, \phi, \psi\rangle, p, k) Q_{1} \in L$, where

$(<B, C, \phi, \psi>, p, k) \in \pi^{*} \times \pi \times\left\{1, \ldots, n_{l e v}\right\}$, and there exists

$<B^{\prime}, C^{\prime}, \phi^{\prime}, \psi^{\prime}>\in\left\{p^{\prime} \mid \operatorname{Int}\left(p, p^{\prime}\right)\right\}$, then

$\left.Q(<B, C, \phi, \psi>, p, k) *\left(<B^{\prime} \wedge C, C^{\prime} \wedge C, \phi^{\prime}(i, \phi(i, \omega)), \psi^{\prime}\right\rangle, p^{\prime}, k+1\right) \in L$.

Every extended plan represents some play history and possible evolution of the play history. The letters lying before "*" represent elementary plans, which the agent has used in the past. The first elementary plan after "*" is a current one, i.e. behaviour of the agent is determined by the $\psi$ map of this plan at present. All other elementary plans are those which the agent is going to execute in the future.

Introduce the notion of the planning function as a map $F: L \times 2^{\pi} \times 2^{\pi} \rightarrow L$. We use the special kind of the planning function defined by the following manner.

Let functions $f: A \rightarrow \pi \cup\{*\}$ and $g: A \rightarrow\left\{0, \ldots, n_{\text {lev }}\right\}$ are projections, $P \in L S_{B} \subseteq \pi, S_{C} \subseteq \pi$ and $Q$ is a letter sequence without "*", possibly empty. Define the planning function $F$, representing the step of our planning system work, by the following clauses:

1. If $P=Q *$ and there exist the largest element $p \in S_{B} \cap D_{1}$ wrt the Prior $_{1}$ relation, then $F\left(P, S_{B}, S_{C}\right)=Q *(p, p, 1)$

2. If following conditions hold:

a) $P=Q * l_{1} \ldots l_{n}$ where $\left.l_{1}=\left(<B_{1}, C_{1}, \phi_{1}, \psi_{1}\right\rangle, p_{1}, k_{1}\right), n \geq 1$;

b) for all $i$ such that $1 \leq i \leq n, f\left(l_{i}\right) \in S_{C}$ and $f\left(l_{i}\right)$ is the largest element of the set $\left(S_{B} \cup\left\{f\left(l_{i}\right)\right\}\right) \cap D_{g\left(l_{i}\right)}$ wrt the $\operatorname{Prior}_{g\left(l_{i}\right)}$ relation;

c) there exists the largest element $p=<B^{\prime}, C^{\prime}, \phi^{\prime}, \psi^{\prime}>\in\left\{p \mid \operatorname{Int}\left(p_{1}, p\right)\right\} \cap S_{B}$ wrt the Prior $_{k_{1}+1}$ relation;

then $F\left(P, S_{B}, S_{C}\right)=Q l_{1} *\left(<B^{\prime} \wedge C_{1}, C^{\prime} \wedge C_{1}, \phi^{\prime}\left(i, \phi_{1}(i, \omega)\right), \psi^{\prime}>, p, k_{1}+1\right)(<$ $\left.C_{1}, C_{1}, \phi_{1}, \psi_{1}>, p_{1}, k_{1}\right) \ldots l_{n}$.

3. If $P=Q * l_{1} \ldots l_{n}$ where $l_{1}=\left(\left\langle B_{1}, C_{1}, \phi_{1}, \psi_{1}\right\rangle, p_{1}, k_{1}\right), n \geq 1$ and there exists $i(1 \leq i \leq n)$ such that:

a) for all $k$ such that $i \leq k \leq n, f\left(l_{k}\right) \in S_{C}$ and $f\left(l_{k}\right)$ is the largest element of $\left(S_{B} \cup\left\{f\left(l_{k}\right)\right\}\right) \cap D_{g\left(l_{k}\right)}$ wrt the $\operatorname{Prior}_{g\left(l_{k}\right)}$ relation;

$b)$ if $i \neq 1$ then $f\left(l_{i-1}\right) \notin S_{C}$ or $f\left(l_{i-1}\right)$ is not the largest element of $\left(S_{B} \cup\right.$ $\left.\left\{f\left(l_{i-1}\right)\right\}\right) \cap D_{g\left(l_{i-1}\right)}$ on the $\operatorname{Prior}_{g\left(l_{i-1}\right)}$ relation;

then $P=Q * l_{1} \ldots l_{i} \ldots l_{n}$. If such $i$ does not exist, then $P=Q l_{1} \ldots l_{n} *$. 
4. For all other cases $F\left(P, S_{B}, S_{C}\right)=P$.

Consider arbitrary history $H$. For every moment $t^{\prime}$ we can built the extended plan by means of the rule: $P(0)=*, P\left(t^{\prime}+1\right)=F\left(P\left(t^{\prime}\right), A p p_{t}{ }^{H}, C o n_{t}{ }^{H}\right)$. Thus, for every time $t$ extended plan can be built by applying consequently the planning function to the initial plan $*$.

\section{How the planning system does work.}

Let us consider one illustrative example. We have the very simple player which has only five basic elementary plans in the basic elementary plan set: (1) "Go to the Ball" ( $\left.p_{1}=<C_{1}, C_{1}, \phi_{1}, \psi_{1}\right\rangle$ ) with the application condition "I see the Ball and I do not possess the Ball", (2) "Intercept of the resting Ball" ( $p_{2}=<C_{2}, C_{2}, \phi_{2}, \psi_{2}>$ ) - "I see the ball and Speed of the Ball is not equal to 0 and I can intercept the Ball before other players and I do not possess the Ball", (3) "Intercept of the moving Ball" ( $\left.p_{3}=<C_{3}, C_{3}, \phi_{3}, \psi_{3}>\right)$ - "I see the Ball and Speed of the Ball is not equal to 0 and $\mathrm{I}$ can intercept the Ball before other players and I do not possess the Ball", (4) "Overtaking" ( $p_{4}=<$ $C_{4}, C_{4}, \phi_{4}, \psi_{4}>$ ) - "There is a player which prevents to the movement", (5) "Kick into the opponent Goal direction" ( $\left.\left.p_{5}=<C_{5}, C_{5}, \phi_{5}, \psi_{5}\right\rangle\right)$ - "I possess the Ball". We suppose for the simplicity that $B_{i} \equiv C_{i}$ for every elementary plan. This entails $\mathrm{App}_{t}{ }^{\mathrm{H}}=\mathrm{Con}_{t}{ }^{H}$ for every moment of time. Thus the behaviour of the agent is determined by such simple skills and the basic elementary plan set $-\pi$ is $\left\{p_{1}, p_{2}, p_{3}, p_{4}, p_{5}\right\}$.

There are two levels in the plan hierarchy: $D_{1}=\left\{p_{1}, p_{2}, p_{3}, p_{5}\right\}$ and $D_{2}=$ $\left\{p_{4}\right\}$. Define Int and Prior $_{1}$ relations as sets of pairs:

$\left\{\operatorname{Int}\left(p_{1}, p_{4}\right), \operatorname{Int}\left(p_{2}, p_{4}\right), \operatorname{Int}\left(p_{3}, p_{4}\right)\right\}$ and $\left\{\operatorname{Prior}_{1}\left(p_{2}, p_{1}\right), \operatorname{Prior}_{1}\left(p_{3}, p_{1}\right)\right\}$, respectively. The Prior $_{2}$ relation is obvious.

At the initial moment the agent behaviour is presented by the extended plan $P_{0}=*$. Suppose that the motionless ball is far from the agent and there is another player which can intercept the ball earlier. In this case $A p p_{0}{ }^{H}=\left\{p_{1}\right\}$ and planner makes extended plan $P_{1}=*\left(p_{1}, p_{1}, 1\right)$ by using rule (1) of the planning function definition. At the time $t_{1}$ our agent have seen the opponent player on its way to the ball. Now $A p p_{t_{1}}{ }^{H}=\left\{p_{1}, p_{4}\right\}$ and $P_{1}$ will be transformed to the plan (see rule (2)) $P_{2}=\left(p_{1}, p_{1}, 1\right) *\left(p_{4}{ }^{*}, p_{4}, 2\right)\left(p_{1}{ }^{*}, p_{1}, 1\right)$.

Here, $p_{1}{ }^{*}$ and $p_{4}{ }^{*}$ are defined by rules (2) and (3) of the definition of $\pi^{*}$, respectively.

Consider two of the possible scenarios. The first - the agent overtakes the opponent player successfully, but still can not intercept the ball before some other player. In this case we have $P_{3}=\left(p_{1}, p_{1}, 1\right)\left(p_{4}{ }^{*}, p_{4}, 2\right) *\left(p_{1}{ }^{*}, p_{1}, 1\right)$.

The second one: the agent believes that it can capture the ball before other players. In this case $A p p_{t_{2}}{ }^{H}=\left\{p_{1}, p_{2}, p_{4}\right\}$. The planning system transforms $P_{2}$ into the extended plan $P_{4}$ by the next two steps (see rules (3) and (1)): $P_{2}^{\prime}=F\left(P_{2}, A p p_{t_{2}}{ }^{H}, A p p_{t_{2}}{ }^{H}\right)=\left(p_{1}, p_{1}, 1\right)\left(p_{4}{ }^{*}, p_{4}, 2\right)\left(p_{1}{ }^{*}, p_{1}, 1\right) *$, $P_{4}=F\left(P_{2}{ }^{\prime}, A p p_{t_{2}}{ }^{H}, A p p_{t_{2}}{ }^{H}\right)=\left(p_{1}, p_{1}, 1\right)\left(p_{4}{ }^{*}, p_{4}, 2\right)\left(p_{1}{ }^{*}, p_{1}, 1\right) *\left(p_{2}, p_{2}, 1\right)$. 
If the plan $P_{4}$ completes successfully at the time $t_{3}$, the agent captures the ball. Then $A p p_{t_{3}}{ }^{H}=\left\{p_{5}\right\}$ and $P_{4}{ }^{\prime}=\left(p_{1}, p_{1}, 1\right)\left(p_{4}{ }^{*}, p_{4}, 2\right)\left(p_{1}{ }^{*}, p_{1}, 1\right)\left(p_{2}, p_{2}, 1\right) *$. $P_{5}=\left(p_{1}, p_{1}, 1\right)\left(p_{4}{ }^{*}, p_{4}, 2\right)\left(p_{1}{ }^{*}, p_{1}, 1\right)\left(p_{2}, p_{2}, 1\right) *\left(p_{5}, p_{5}, 1\right)$.

\section{Conclusion}

Our approach is somewhat analogous to that presented in $[1,2]$, with one essential difference: our planning system works on-line, and plans refinements are being made dynamically in case of need. More detailed comparisons deserve the further investigations and are to be presented elsewhere. The further development of the method itself and the more precise formulation of its essence is a goal of further work.

\section{References}

1. M. Ginsberg. Modality and Interrupts. Journal of Automated Reasoning (14) 1 (1995) pp. 43-91.

2. M. Ginsberg, H. Holbrook. What defaults can do that hierarchies can't. Fundamental Informaticae (21) (1994) pp. 149-159. 